\title{
Unsteady Tangent Hyperbolic Fluid on Radiated Exponentially Porous Surface
}

\author{
Santhosh H.B, M. Karuna Prasad , C.S.K. Raju, Mahesha
}

\begin{abstract}
This analysis refers the radiation and porosity effects on unsteady hyperbolic tangent fluid over exponentially stretching sheet. Using similarity transformation the governing equations which are partial differential equations in nature have been modified into nonlinear differential equations $(\mathrm{ODE})$, and then we obtained the solution by shooting technique along with Runge-Kutta method. The dimensional less velocity and temperature have been represented graphically. We have found local Nusselt number and friction factor for distant dimensional less physical parameter values, and they displayed in table. At the end of the analysis we conclude that magnetic field decreases velocity of hyper tangent fluid and porosity effect decreases velocity of hyper tangent fluid. Further the radiation enriches temperature profile in injection case than suction case. This conclusion tells us injection case is useful in temperature transportation than suction case.
\end{abstract}

Keywords: ODE, dimensional less velocity.

\section{INTRODUCTION:}

The non-Newtonian fluid in which constitutive equation is holds true for high and low shear rate, this type of fluid is called tangent hyperbolic fluid. This fluid explains blood flows and physical behaviour of the blood very accurately. Because of these views many researchers discussed peristaltic flow by assuming tangent hyperbolic fluid as a model fluid for blood. The heat transfer analysis has been investigated in wider range due to its many advantages in geothermal systems, energy preserve units, heat insulation and heat exchangers.

By the motivations above, this study describes unsteady magnetic and dissipative tangent hyperbolic fluid due to radiated exponentially porous surface.
Revised Manuscript Received on December 15, 2019

Santhosh H.B, Mathematics Department, GITAM (Deemed To Be University), Bengaluru-562163, Karnataka, India. santu1603@gmail.com M. Karuna Prasad, Mathematics Department, GITAM (Deemed To Be University), Bengaluru-562163, Karnataka, India. karunaprasad9@gmail.com

C.S.K. Raju, Mathematics Department, GITAM (Deemed To Be University), Bengaluru-562163, Karnataka, India. sivaphd90@ gmail.com Mahesha, Mathematics Department, U.B.D.T. College of Engineering Davangere-577004, Karnataka, India. maheshubdt@gmail.com
The researcher Nadeem et al. [1] discussed Magnetohydrodynamic peristaltic hyperbolic tangent fluid model in a downward asymmetric canal subjected to low negligible large wavelength and approximations. Numerical study on nonlinear nature of boundary layer fluid flow and temperature transfer of tangent hyperbolic fluid with an incompressible nature has been studied by Gaffar et al. [2] and concluded that velocity and temperature profiles are increased by raising the values of Biot number. Numerical study on diffusive magnetohydrodynamic tangent hyperbolic fluid on a stretching cylinder was explored by Reddy et al. [3] and observed that magnetic field enriches temperature profile and velocity slip parameter reduces velocity profiles. Choudhary et al. [4] analysed the theoretical investigation on uniform magnetic field influence on unsteady flow of incompressible electrically viscous conducting fluid over a stretching permeable sheet. Hussin et al. [5] explored on viscous dissipation and connective conditions influence on magnetohydrodynamic tangent hyperbolic fluid on a non-linear stretching surface and they compared the values of local Nusselt and friction factor number between numerical method and analytical method. Mathematical modeling using spectral relaxation method on 2-dimensional fluid flow of mass and heat transport of hyperbolic tangent fluid with thermal dispersion has been carried out by Rao and Rao et al. [6], and conclude that thermal dispersion strongly influence on temperature profile. Jyothi et al. [7] discussed about peristaltic transportation of tangent hyperbolic fluid in two-dimensional symmetric canal under assumption of negligible Reynolds number with high wave length. Kumar et al. [8] addressed on numerical investigation on hyperbolic tangent fluid over sensor surface and unstable thermal conductivity. The non-linear boundary layer flow of hyperbolic tangent incompressible nonNewtonian fluid over erect porous plate has been analyzed by Prasad et al. [9]. The effect of thermal generation and absorption in twice stratified MHD hyperbolic tangent incompressible fluid over stretching cylinder implanted in a porous media under immersion of nanoparticles has been studied by Nagedramma et al. [10]. Recent studies have been given in [11-12].

\section{FORMULATION OF PROBLEM:}

We considered MHD unsteady boundary layer flow of a hyperbolic tangent fluid on permeable exponentially stretching/shrinking sheet. The stretching surface is supposed to assume in direction of $x$ - axis with exponential stretching velocity $U=U_{0} e^{N x / L} /(1-c t)$, and subjected to magnetic field $B$ in $y$ direction. The flow is subjected in positive vertical direction, and this is normal to

\section{Published By:}

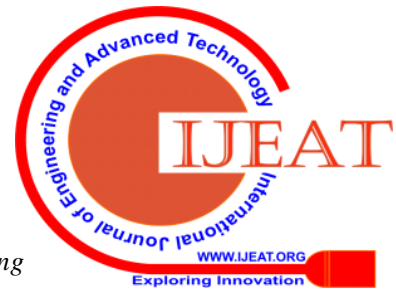


$x-$ axis. The temperature maintained near the surface is $T=T_{\infty}+T_{0} e^{N x / 2 L} /(1-c t)$.

$S=A\left\{\left(\mu_{0}+\mu_{\infty}\right) \tanh (\tau \overline{\dot{\gamma}})^{n}+\mu_{\infty}\right\}$

Where $\mu_{0}$ zero shear rate viscosity, $\mu_{\infty}$ the large shear rate viscosity, $\tau$ is the time related constant, $n$ power law of index and $\overline{\dot{\gamma}}$ is given by,

$\overline{\dot{\gamma}}=\sqrt{1 / 2 \operatorname{tr}\left(A_{1}^{2}\right)}$

Assuming that $\mu_{\infty}=0$ is assimilated in extra stress tensor $S$ , further as hyperbolic tangent fluid nominates the shear thinning spectacles, thus $\tau \overline{\dot{\gamma}}<1$. After using these above mentioned hypotheses in equation (1); it takes the form as follows.

$S=\mu_{0}\left[(\tau \overline{\dot{\gamma}})^{n}\right] A_{1}$

After replacement the above equation converts the following form:

$$
S=\mu_{0}\left[1+n(\tau \overline{\dot{\gamma}}-1)^{n}\right] A_{1}
$$

With the reference to above, we have come across the following governing equations.

$$
\frac{\partial u}{\partial y}=-\frac{\partial u}{\partial x}
$$

$$
u \frac{\partial u}{\partial x}+v \frac{\partial u}{\partial y}+\frac{\partial u}{\partial t}=-v_{f}(n-1) \frac{\partial^{2} u}{\partial y^{2}}+\tau m \sqrt{2}\left(\frac{\partial u}{\partial v}\right)\left(\frac{\partial^{2} u}{\partial y^{2}}\right)-u \frac{\sigma B_{0}^{2}}{\rho}+\left[g \beta_{T}\left(T-T_{\infty}\right)\right]-\frac{u}{K_{0}} v
$$

The expression for an extra stress tensor $S$ of hyperbolic tangent fluid is as follows.

$$
u \frac{\partial T}{\partial x}+v \frac{\partial T}{\partial y}+\frac{\partial T}{\partial t}=\frac{k}{\rho C_{p}} \frac{\partial^{2} T}{\partial y^{2}}+\frac{16 \sigma^{*} T_{\infty}^{3}}{3 \rho C_{p} k^{*}} \frac{\partial^{2} T}{\partial y^{2}}+\frac{v}{C_{p}}(1-n)\left(\frac{\partial u}{\partial y}\right)^{2}+\frac{u n}{C_{p} \sqrt{2}}\left(\frac{\partial u}{\partial y}\right)\left(\frac{\partial u}{\partial y}\right)^{2}
$$

The subjected boundary limitations are

at; $y=0, U=u, v=V(x), T_{w}=T$, $u \rightarrow 0, T \rightarrow T_{\infty}$, as $; y \rightarrow \infty$

(8) To transform the above equations into ODE, we required the below transformation as,

$$
\begin{aligned}
& u=\frac{U_{0}}{(1-c t)} e^{N k / L} f^{\prime}(\eta), v=-N\left[v_{f} U_{0} / 2 L(1-c t)\right]^{1 / 2} e^{N k / 2 L}\left[f(\eta)+\eta f^{\prime}(\eta)\right] \\
& \eta=\left[U_{0} / 2 L v_{f}(1-c t)\right]^{1 / 2} e^{N k / 2 L} y, T-T_{\infty}=T_{0} e^{N k / 2 L} \theta(\eta) /(1-c t),
\end{aligned}
$$

By applying equation (10) into equations (5) is automatically satisfies the continuity. Equations (6) and (7) are transformed as follows.

$\left\{(1-n)+n W e f^{\prime \prime}\right\} f^{\prime \prime \prime}-N\left\{f^{\prime 2}-f f^{\prime \prime}\right\}+2 G r \theta-f^{\prime}(2 A+M+K)-A f^{\prime \prime}=0$

$\left(1+\frac{4}{3} R_{a}\right) \theta^{\prime \prime}-N\left\{f \theta-f^{\prime} \theta\right\}-(n-1) E c \operatorname{Pr} f^{\prime \prime 2}+n W e E c \operatorname{Pr} f^{\prime \prime 3}+A(\theta+\theta)=0$ (12) The reformed boundary

constraints are

$f(\eta)=S, f^{\prime}(\eta)=1, \theta(\eta)=1$ at; $\eta=0$
$f^{\prime}(\eta) \rightarrow 0, \theta(\eta) \rightarrow 0 a t ; \eta \rightarrow \infty$

The skin friction coefficient $(C f)$, and local Nusselt number $(\mathrm{Nu})$ are as follows

$C f \operatorname{Re}^{1 / 2}=f^{\prime \prime}(0)$

$N u \operatorname{Re}^{-1 / 2}=-\left(1+\frac{4}{3} R\right) \theta^{\prime}(0)$, 
(14)

\section{RESULT AND DISCUSSIONS:}

The equations (11)-(12) along with limitation for boundary given in (13) have been solved by shooting technique with Runge Kutta method. In the

way to acquire the results, we assumed dimensionless param eters as;
Generally increasing the values of porosity gives us to increasing voids on the surface, because of increasing voids the velocity of fluid slows down. Dig.7 shows the temperature in injection cases increases with porosity. It is clear that, the temperature in suction profiles is more than the temperature in injection profile. Magnetic field effect on both temperature and velocity profile have been given in Digs 8-9, from these we identified that, the velocity profiles are increased by magnetic parameter. This due to fact that electric conducting fluid in the presence of magnetic field induces a retarding Lorentz force which works in perpendicular direction to the applied magnetic field. Because of this continuous retardation the velocity profiles are reduced.

\section{CONCLUSION}

$N=0.2, G r=0.2, G c=0.2, A=0.2, R=0.2, K=0.5, S c=1, n=0.2, M$ w considered the magnetic field and porosity on $W e=0.3, E c=0.3$ these values are fixed values in overall of this analysis expect some changes in corresponding graphs and tables. Injection profiles in the graph are represented by solid lines, suction profiles are represented by dashed lines.

Digs. 2 and 3 indicates the effect of radiation on temperature and velocity profile, by these diagrams we concluded that radiation enriches both velocity and temperature profile. As radiation develops heat molecules, these molecules boost up the velocity and temperature of boundary layer. Compare to velocity in suction case the velocity in injection case gains higher values. This difference is due to the fact that, in suction case the molecules are absorbed due to suction process due to this the velocity of molecules slows down. Digs. 4-5 indicates the influence on unsteady parameter on both temperature and velocity profile, from these graphs we concluded that, the unsteady parameter increases the velocity and temperature profile. Compare to velocity and temperature in suction case the velocity in injection case gains higher values. Digs. 6-7 indicates the porosity effect on both velocity and temperature profile. Dig-6 shows increasing the values of porosity parameter decreases velocity profiles, this graph clarifies the velocity in injection case is lesser than the velocity in suction case. unsteady hyperbolic tangent fluid over exponentially stretching sheet. Using similarity transformations the arising set of governingpartial differential equations have been transformed into differential equations (ODE) with non linear nature. and then we obtained the solution by RungeKutta with shooting technique.. The main out puts of our analysis are mentioned below.

- The magnetic field enhances boundary layer velocity.

- The effect of radiation increases both velocity and temperature of boundary layer.

- Hiking the values of magnetic field decrements both heat transfer rate and friction between the particles.

- Increasing of radiations decrements heat transfer rate in both suction and injection case.

Table 1: Skin friction $\left(C_{f}\right)$ and local Nusselt number $\left(N u_{x}\right)$ by the influence of various dimensional less parameters of both suction and injection and suction case.

\begin{tabular}{|c|c|c|c|c|c|c|c|}
\hline$R$ & $A$ & $K$ & $M$ & \multicolumn{2}{|c|}{$C_{f}$} & \multicolumn{2}{c|}{$N u_{x}$} \\
\hline & & & & Injection & Suction & Injection & Suction \\
\hline 1 & & & & 0.740779 & 0.726904 & 2.131026 & 2.131734 \\
\hline 1.1 & & & & 0.741397 & 0.727352 & 2.108361 & 2.119604 \\
\hline 1.2 & & & & 0.741931 & 0.727739 & 2.090826 & 2.111842 \\
\hline & 0.01 & & & 0.727818 & 0.728814 & 1.443222 & 1.483576 \\
\hline
\end{tabular}


Unsteady Tangent Hyperbolic Fluid on Radiated Exponentially Porous Surface

\begin{tabular}{|l|l|l|l|l|l|l|l|}
\hline & 0.015 & & & 0.727580 & 0.728568 & 1.444277 & 1.485470 \\
\hline & 0.02 & & & 0.727346 & 0.728325 & 1.445244 & 1.487319 \\
\hline & & 0.1 & & 0.764708 & 0.766301 & -1.309914 & -1.461556 \\
\hline & & 0.5 & & 0.749933 & 0.751663 & -1.207299 & -1.367159 \\
\hline & & 1 & & 0.734577 & 0.736429 & -1.210352 & -1.383104 \\
\hline & & & 1 & 0.747520 & 0.749254 & -0.983110 & -1.130063 \\
\hline & & & 2 & 0.719997 & 0.721918 & -1.169112 & -1.343352 \\
\hline & & & 3 & 0.698142 & 0.700166 & -1.567202 & -1.769522 \\
\hline
\end{tabular}

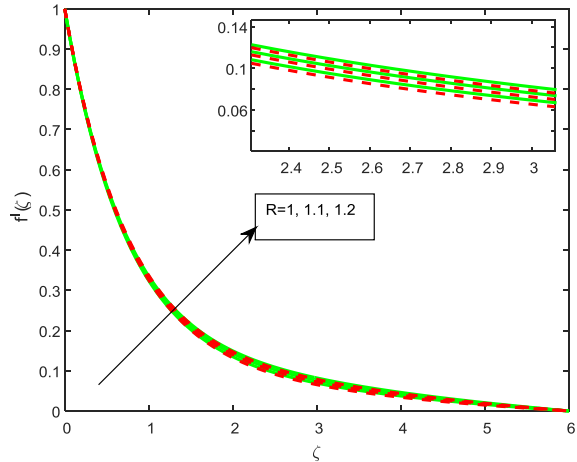

Dig. 2 nature of $f$ by $R$

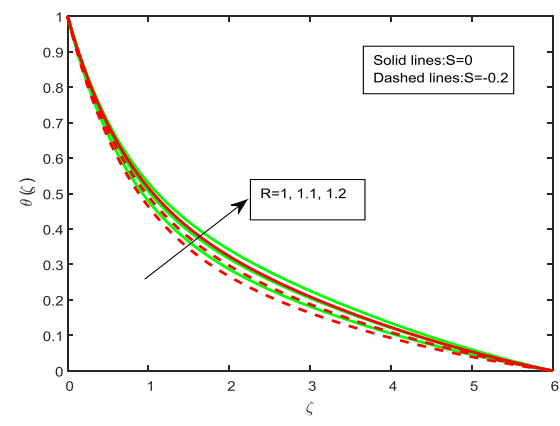

Dig. 3 nature of $\theta$ by $R$

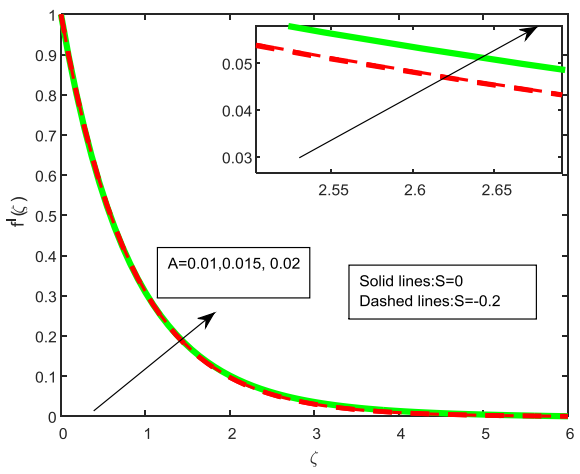

Dig. 4 nature of $f$ by $A$

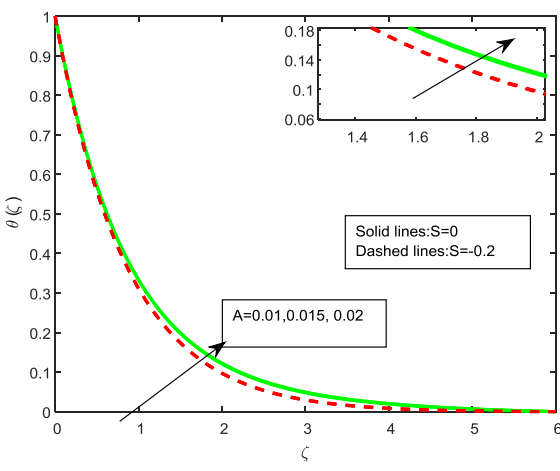

Dig. 5 nature of $\theta$ by $A$.

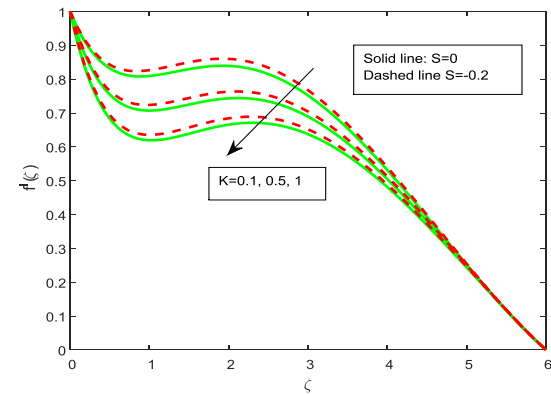

Dig. 6 nature of $f$ by $K$

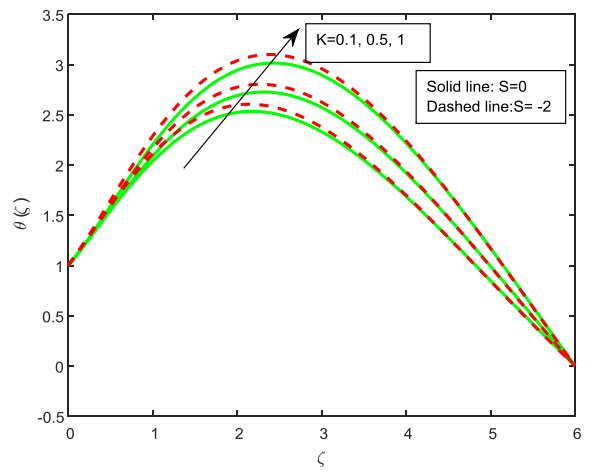

Dig. 7 nature of $\theta$ by $A$ 


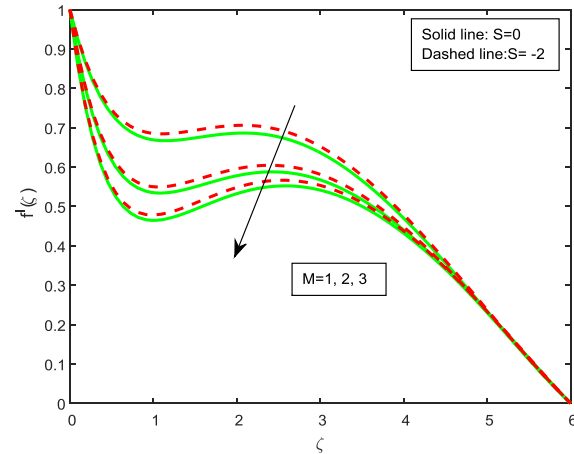

Dig. 8 nature of $f$ by $M$.

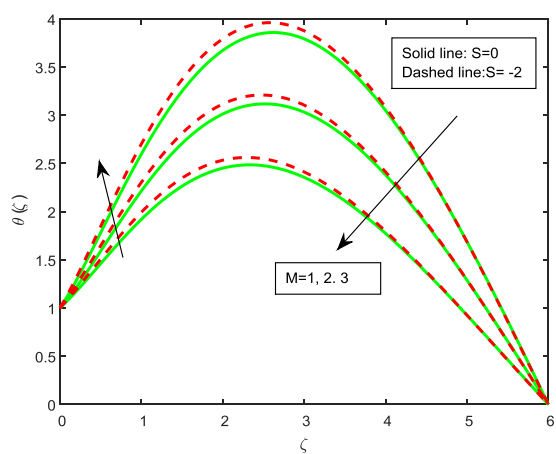

Dig. 9 nature of $\theta$ by $M$.

\section{REFERENCE}

1. S. Nadeem, S. Akram, Magnetohydrodynamic peristaltic flow of a hyperbolic tangent fluid in a vertical asymmetric channel with heat transfer, Acta Mech. Sin. Xuebao. 27 (2011) 237-250.

2. S.A. Gaffar, V.R. Prasad, O.A. Béeg, Numerical study of flow and heat transfer of non-Newtonian Tangent Hyperbolic fluid from a sphere with Biot number effects, Alexandria Eng. J. 54 (2015) 829841.

3. M. Gnaneswara Reddy, J. Manjula, P. Padma, Mass Transfer Flow of MHD Radiative Tangent Hyperbolic Fluid over a Cylinder: A Numerical Study, Int. J. Appl. Comput. Math. 3 (2017) 447-472.

4. M.K. Choudhary, S. Chaudhary, R. Sharma, Unsteady MHD Flow and Heat Transfer over a Stretching Permeable Surface with Suction or Injection, Procedia Eng. 127 (2015) 703-710.

5. A. Hussain, M.Y. Malik, T. Salahuddin, A. Rubab, M. Khan, Effects of viscous dissipation on MHD tangent hyperbolic fluid over a nonlinear stretching sheet with convective boundary conditions, Results Phys. 7 (2017) 3502-3509.

6. K. Uma, G. Koteswara, Boundary Layer Flow Of MHD Tangen Hyperbolic Fluid Past A Vertical Plate In The Presence Of Thermal Dispersion Using Spectral Relaxation Method, (2017) 28-41.

7. S. Jyothi, M.V.S. Reddy, P. Gangavathi, Hyperbolic Tangent Fluid Flow Through A Porous Medium in an Inclined Channel with Peristalsis, 1 (2016) 113-121

8. K. Ganesh Kumar, B.J. Gireesha, M.R. Krishanamurthy, N.G Rudraswamy, An unsteady squeezed flow of a tangent hyperbolic fluid over a sensor surface in the presence of variable thermal conductivity, Results Phys. 7 (2017) 3031-3036.

9. V. Ramachandra Prasad, S. Abdul Gaffar, O. Anwar Beg, Free convection flow and heat transfer of Tangent Hyperbolic past a vertical porous plate with partial slip, J. Appl. Fluid Mech. 9 (2016) 1667-1678.

10. Nagendramma, V., Leelarathnam, A., Raju, C. S. K., Shehzad, S. A., \& Hussain, T. (2018). Doubly stratified MHD tangent hyperbolic nanofluid flow due to permeable stretched cylinder. Results in Physics, 9, 23-32.

11. Mamatha Patil, Mahesha, C.S.K.Raju, Heat and mass transfer in MHD tangent hyperbolic fluid over chemically reacting exponentially stretching surface with porous medium. Journal of Nano fluids, 7 (5) , 853-861.

12. Mamatha Patil, Mahesha, C.S.K.Raju, Convective conditions and dissipation on Tangent Hyperbolic fluid over a chemically heating exponentially porous sheet. Journal of Non-linear Engineering, DOI 10.1515/nleng-2018-0003

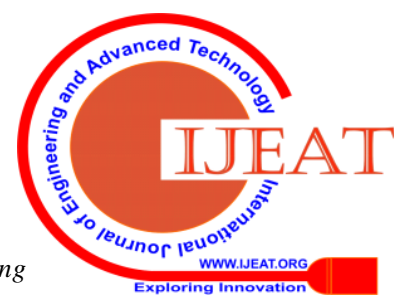

